\title{
Introduction to inaugural issue from PIAM
}

\author{
Cynthia Wirth $^{1} \cdot$ Martin Schäfer ${ }^{2}$
}

Published online: 1 June 2016

(c) Springer International Publishing Switzerland 2016

Progress in Additive Manufacturing has been initiated and inspired due to a crescent demand from both industrial and scientific communities to jointly contribute with their new achievements and applied results in the different areas of these technologies. As an interdisciplinary area, AM interconnects concepts from material science, simulation, manufacturing, tooling and design in a broad field of applications. Although each of these areas are well represented by highly scored journals, which can figure as platform for publication of their achievements, Progress in Additive Manufacturing urge to unite scientific and industrial papers, reviews and short communications within the scope of one single journal.

One singular aspect from AM is related with the velocity with which AM technologies have been inserted in industry, laboratories and research centers. Nowadays, it can be certainly expected to find a "3D printer" in almost every technological laboratory in every major manufacturing industry or research group worldwide. AM technologies have been accepted and then implemented prior to any other aspect of the technology development. The design rules, or better the freedom in design rules, have been pushing designers over their borders. Material scientists are being forced to understand that material can be manipulated locally or layer-by-layer to achieve new properties and that the opportunities to create graded materials are

Cynthia Wirth

cynthia.wirth@siemens.com

1 Siemens AG, Power and Gas Division, Large Gas Turbine, Huttenstr. 12, 10553 Berlin, Germany

2 Siemens AG, Corporate Technology, Research and Technology Center, Siemensdamm 50, 13629 Berlin, Germany extended. Even the industry has implemented AM technologies prior to their standards and are urging now for that, therefore quality assurance are examined to address industry demands/requirements.

By facing the fact that $\mathrm{AM}$ is pushing us beyond the conventional boundaries of science and technology, we have agreed to create PIAM as platform for all unconventional and re-created aspects of material science, manufacturing, tooling, design, simulation and standardization.

We are very glad that PIAM has been well accepted by the scientific and industrial communities. The first issue in a double edition, just reinforces this fact. In the first edition, the three different classes of materials have been explored, metals, ceramics and polymers. Also the most wide-spread AM technologies are present, powder bed fusion, material extrusion and material jetting. The articles increase the understanding of the correlation and dependency between materials, processes and part quality. They comprehend aspects of the process chain qualification and material characteristics especially for the powder bed technology and the material extrusion and jetting.

Additionally the design is shown as an important tool for the use of AM particularly for production. The relevance of the additive technologies for industrial processes is described on background of traditional casting and hybrid manufacturing. The investigations present current developments of technologies and the optimization of processes e.g. by parameter modeling and indicate an important view on the research activities and also on industrial utilization of AM.

As editors, we are very thankful for the support of all colleagues and contributors. To the authors, representing the research community all over the world, we really appreciate their work and cooperation to generate an interesting journal on a high scientific level! 